\title{
Resistant Hypertension - A Fourth Cause
}

\author{
K Powell, Dr K S Myint \\ Department of Endocrinology
}

\section{Background}

Resistant Hypertension is defined as blood pressure which remains above the goal despite treatment with at least three antihypertensive agents. Because of much higher cardiovascular risk, further evaluation of secondary cause is crucial in these cases. The most common causes are renal artery stenosis, Conn's syndrome and pheochromocytoma.

\section{Case 1}

68 year old gentleman, wheelchair bound, 12 year history of hypertension with BP persistently above 200/100,

\section{Past Medical History}

- Stroke resulting in partial sightedness and wheelchair bound

- Obstructive sleep apnoea

- Left Ventricular Hypertrophy

- Type 2 diabetes mellitus

- Cervical spondylosis with chronic pain - Psoriasis

\section{Drug History}

At presentation he was on 15 different medications including

- Doxasozin 8mg

- Nifedipine MR 30mg

- Lisinopril 20mg

- Propranolol 160mg

- Furosemide $40 \mathrm{mg}$

All of which patient confirmed he was taking regularly.

\section{Investigations}

Ultrasound kidneys - normal

Urinary normetanephrines

Urinary metanephrines

Plasma renin

Plasma aldosterone

24hr BP

Mean daytime $B P$

Mean nocturnal BF

\section{$\mathbf{P}$} gress and Outcome

Despite 3 years of adjusting antihypertensive therapy, BP was persistently above 190 systolic. Renal denervation therapy was considered. Urine was sent for analysis with HPLC-MS to Sandwell laboratory in Birmingham. No antihypertensives were identified.

Patient was recalled and reviewed and the issue of adherence was gently and sensitively discussed. Patient admitted hiding tablets as he could not cope with the amount of medication. All medication was rationalised. Antihypertensives were reduced to spironolactone and amlodipine only,

$\mathrm{BP}$ was persistently controlled at average $130 / 78 \mathrm{mmHa} 2$ years after this intervention

\section{Discussion}

Adherence to medical therapy is the major issue in managing hypertension. Polypharmacy, potential side effects of medication and asymptomatic nature of hypertension off treatment are major contributory factors. The newer assay of urinary analysis for all antihypertensive screening has become increasingly useful for identifying potential issues of adherence to medical therapy and can be an aid to initiate discussion. It is essential to involve the patient in the aareement of their manadement plan.

\section{Objective}

We present two cases of resistant hypertension where we faced therapeutic challenges. Secondary causes were not established but eventually the reason was identified as not adhering to medication.

\section{Case 2}

47 year old wheelchair bound gentleman was referred with resistant hypertension despite multiple antihypertensive therapy. BP 168/90
Past Medical History

- Glomerulonephritis

- Ischaemic heart disease with recurfrent angina

- Previous myocardial infarction

- Recurrent stroke including an intracranial bleed

- Postg stroke epilepsy
Drug History

16 different medications including

- Bendroflumethiazide $2.5 \mathrm{mg}$

- Amlodipine $10 \mathrm{mg}$

- Lisinopril 40mg

- Candesartan 32mg

- Spironolactone 50mg

- Doxazsin 8mg

- Propranolol 320mg

\section{Investigations}

MRA renal arteries - no renal artery stenosis
Plasma metanephrines Plasma normatenaphrines

Plasma renin

Plasma aldosterone

24hr BP

Mean daytime $B P$

Mean nocturnal $\mathrm{BP}$
$251(80-510) \mathrm{pmol} / \mathrm{L}$ $366(120-1180) \mathrm{pmol} / \mathrm{L}$

$50 \mathrm{mU} / \mathrm{L}$

$151 \mathrm{pmol} / \mathrm{L}$

$154 / 101 \mathrm{mmHg}$ $159 / 101 \mathrm{mmHg}$

\section{Progress and Outcome}

Patient was repeatedly admitted to hospital with strokes and unstable angina. His wife became very unhappy if potential issue of poor adherence to therapy was raised. Team was concerned in case there was a potential safeguarding issue. Urine was sent to Sandwell laboratory at Birmingham for analysis of antihypertensives. Only hydralazine was identified among all antihypertensives the patient was supposed to be taking.

Patient was recalled and issue was sensitively discussed. Patient admitted hiding medication without his wife's knowledge due to unbearable side effects. All medication was rationalised. Antihypertensives were reduced to amlodipine only. BP was persistently controlled at average $120 / 85 \mathrm{mmHg} 2$ years after this intervention.

\section{Acknowledgement}

Sandwell laboratory, Birmingham

Further information: Swe Myint khinswe.myint@nnuh.nhs.uk 